\title{
MIGRACIÓN, URBANIZACIÓN Y DESARROLLO
}

\author{
ANDREI ROGERS \\ Coordinador del Area de Asentamientos Humanos y Servicios \\ International Institute for Applied Systems Analysis
}

INTRODUCCIÓN: perspectivas del problema

LA CRECIENTE interdependencia económica global, el aumento de la competencia por reducir las reservas de los recursos y las disparidades, cada vez más grandes en el bienestar material, han ocasionado que el futuro crecimiento de la población sea un tema obligado a tratar en los asuntos internacionales. El crecimiento acelerado de la población conlleva consecuencias muy importantes en lo social, económico y político. Afecta los niveles de salud pública y bienestar, así como la calidad del ambiente de los habitantes de ese lugar. Algunas de estas consecuencias no son muy comprensibles, a pesar de que se sabe que reducir tal crecimiento no puede resolver sino sólo mitigar, los diversos problemas asociados con el crecimiento demoeconómico.

El objetivo principal del Area de Asentamientos Humanos y Servicios del Instituto Internacional para el Análisis de Sistemas Aplicados (IIASA), se relaciona con el desarrollo de mejoras en los métodos para analizar los determinantes y consecuencias del crecimiento demoeconómico, así como de la comprensión de temas fundamentales y las opciones asociadas con éste. En este trabajo se revisan y resumen los esfuerzos más recientes que ha efectuado el Área, con el objeto de contribuir a un análisis interdisciplinario de los problemas y a la comprensión multidimensional (sistemas) de las alternativas estratégicas disponibles.

\section{Desarrollo urbano}

La población mundial, en 1980 , ascendía a 4,300 millones de habitantes aproximadamente y revelaba una tasa de crecimiento aproximado del 1.8 por ciento anual. Según dicha tasa de crecimiento, al final de este siglo la población mundial ascenderá a 6,300 millones de habitantes. Sin embargo, el descenso reciente de las tasas de fecundidad en muchos países de menor desarrollo, ha llevado a los demógrafos a proyectar una cifra más baja, aproximadamente 6,000 millones para el año 2000, y una "franja" amplia que va de los 8,000 millones a 10,500 millones para el año 2050. 
Gráfica 1

CRECIMIENTO DE LA POBLACIÓN URBANA Y RURAL EN EL MUNDO, 1800-2000 (EN MILLONES)



El crecimiento de la población urbana ha sido todavía más explosivo (gráfica 1). En la actualidad, cerca de 1,800 millones de personas -el 42 por ciento de la población mundial- viven en áreas urbanas y esta cifra aumenta a un ritmo de casi 3 por ciento anual. En el comienzo del siglo pasado, la población urbana mundial ascendía a un total de 25,000 millones. Los cálculos de las Naciones Unidas deducen que cerca de 3,100 millones de personas, casi dos veces el total de la población urbana de la actualidad, vivirán en áreas urbanas en el año 2000 (Naciones Unidas, 1976), es decir, que la cifra se habrá multiplicado 124 veces en dos siglos.

Las tasas de crecimiento son todavía más dramáticas a nivel de cada asentamiento urbano. El cuadro 1 muestra las proyecciones más recientes de las Naciones Unidas respecto del crecimiento de algunos de los centros urbanos más grandes del mundo en países de menor desarrollo, en donde se indica que el tamaño del multiplicador del crecimiento de la población -el momentum urbano- de algunas ciudades es verdaderamente pavoroso. Por ejemplo, durante los 25 años entre 1975 y el año 2000, se espera que las ciudades de Lima, México, Jakarta y Teherán triplicarán su población; se proyecta que San Pablo y Seúl tendrán un crecimiento correspondiente a un factor de 2, y que Addis Abeba, Nairobi, Lagos y Kinshasa aumentarán su población cuatro veces. Se proyecta que la ciudad de México será 
la ciudad más poblada, con más de 31 millones de habitantes. Le seguirá San Pablo con cerca de 26 millones (Naciones Unidas, 1976).

\section{Problemas urbanos}

Al igual que el crecimiento acelerado de la población, el crecimiento urbano acelerado aumenta las dificultades para proporcionar a la población el sustento, empleos, servicios e infraestructura necesarios. El incremento acelerado de la población urbana ocasiona que se excedan los presupuestos en salud y educación, limita la posibilidad de reducir los niveles de desempleo, y exacerba los problemas relacionados con el aprovisionamiento de vivienda adecuada, abastecimiento de alimentos, energía, transporte, agua y sanidad. La "inversión demográfica" necesaria exclusivamente para mantener los niveles actuales en muchas áreas de urbanización acelerada, tendrá que duplicarse o triplicarse en los próximos veinticinco años. El hecho de que estas áreas se encuentren en la mayoría de los países que tienen menor capacidad para enfrentar tal inversión, aumenta las dificultades que se asocian al agravamiento de la capacidad utilizada de las ciudades en los países de menor desarrollo.

CuAdro 1

Cifras CAlCuladas y PROYECCIONES de la POBLACión EN LAS QUINCE CIUDADES MÁS HABITADAS

\begin{tabular}{|c|c|c|c|c|c|}
\hline \multirow{2}{*}{ Ciudad } & \multicolumn{3}{|c|}{ Poblacion (en millones) } & \multicolumn{2}{|c|}{$\begin{array}{l}\text { Incremento multiple } \\
\text { sobre la base anual }\end{array}$} \\
\hline & 1950 & 1975 & 2000 & $1950-1975$ & $1975-2000$ \\
\hline Cairo, Egipto & 2.4 & 6.9 & 16.4 & 2.9 & 2.4 \\
\hline Addis Abeba, Etiopia & 0.2 & 1.1 & 4.2 & 4.8 & 3.9 \\
\hline Nairobi, Kenia & 0.1 & 0.7 & 3.4 & 5.5 & 4.5 \\
\hline Lagos, Nigeria & 2.9 & 2.1 & 9.4 & 7.2 & 4.6 \\
\hline Kinshasa, Zaire & 0.2 & 2.0 & 9.1 & 12.5 & 4,4 \\
\hline Cd. México, México & 2.9 & 10.9 & 31.6 & 3.8 & 2.9 \\
\hline Sao Paulo, Brasil & 2.4 & 10.0 & 26.0 & 4.1 & 2.6 \\
\hline Bogots, Colombia & 0.7 & 3.4 & 9.5 & 5.2 & 2.8 \\
\hline Guayaquil, Ecuador & 0.3 & 1.0 & 3.1 & 4.0 & 3.1 \\
\hline Iima, Perú & 0.6 & 3.9 & 12.1 & 6.4 & 3.1 \\
\hline Jakarta, Indonesia & 1.6 & 5.6 & 16.9 & 3.6 & 3.0 \\
\hline Terăn, Irán & 1.0 & 4.4 & 13.8 & 4.3 & 3.1 \\
\hline Seul, Corea & 1.0 & 7.3 & 18.7 & 7.1 & 2.6 \\
\hline Karachi, Paquistán & 1.0 & 4.5 & 15.9 & 4.3 & 3.6 \\
\hline Bangkok, Tailandia & 1.0 & 3.3 & 11.0 & 3.4 & 3.4 \\
\hline
\end{tabular}

FUENTE: Naciones Unidas (1976: 77-82) 
BARRIADA Y ASENTAMIENTOS INCONTROLABLES. POR CIENTO DEL TOTAL DE LA POBLACIÓN EN CIUDADES (CERCA DE 1960-1970)

\begin{tabular}{lcc}
\hline Ciudad, pais & 8 & $\begin{array}{c}1970 \\
\text { (US.\$) }\end{array}$ \\
\hline Caracas, Venezuela & 980 \\
Cd. México, México & 40 & 670 \\
Lusaca, Zambia & 46 & 400 \\
Bogota, Colombia & 58 & 340 \\
Nairobi, Kenia & 60 & 150 \\
Kinshasa, zaire & 33 & 90 \\
Manila, Filipinas & 60 & 210 \\
\hline
\end{tabular}

FUENTE: Sinclair (1978).

Aproximadamente, una tercera parte de la población urbana en la mayoría de los países de menor desarrollo vive en asentamientos con acceso deficiente al agua, alcantarillado, transporte, sanidad, educación y vivienda, y casi un 60 por ciento vive, en algunos países, en asentamientos ilegales (cuadro 2).

\section{Conclusión}

Las tasas elevadas de crecimiento urbano y el aumento del consumo que se deriva del aumento del ingreso per cápita, (que continúa a una tasa anual de 4 a 5 por ciento), significa una tasa de crecimiento anual del ingreso total urbano y una demanda de bienes y servicios de aproximadamente 9 por ciento, duplicándose esta cantidad cada siete $u$ ocho años.

Un examen del panorama futuro en relación al crecimiento de la población y urbanización mundiales, revela, en forma contundente, que los desarrollos históricos paralelos que se combinaron para crear los problemas de los asentamientos humanos en la actualidad, continuarán en algunas partes del mundo durante el resto de este siglo y principios del siguiente. Sin embargo, el número de habitantes en el mundo continuará ascendiendo en un futuro cercano, así como la proporción de habitantes que viva en asentamientos urbanos. Es probable que la población de los centros urbanos continúe en ascenso a una tasa alarmante, en especial la de las aglomeraciones urbanas más grandes de los países de menor desarrollo. Los problemas que se han originado por esta transformación son muchos e involucran costos privados y sociales muy elevados. Es importante tener en mente, que también existen beneficios evidentes uuando se toman en consideración las políticas de intervención en el proceso de urbanización. La mejor comprensión de la dinámica y consecuencias del crecimiento poblacional 
rural-urbano, así como el desarrollo económico, representan un ingrediente esencial de tales consideraciones, además del punto central en el proceso de cambio, junto con sus manifestaciones. El objetivo principal del Área de Asentamientos Humanos y Servicios consiste en lograr una mayor comprensión en la investigación sobre migración, urbanización y desarrollo.

\section{Perspectivas de investigación: análisis de los determinantes y consecuencias}

Un connotado profesor de la Universidad de Harvard acostumbraba decir a sus estudiantes que la diferencia entre la economía y la sociología es muy simple. La economía es el estudio de cómo el hombre efectúa sus elecciones. La sociología se refiere al porqué no tiene ninguna elección por hacer.

Con los principios de la sociología y las ciencias actuariales, no es de sorprender que la perspectiva de investigación de los demógrafos recaiga principalmente en el método de descomposición. La contribución de las diversas causas y el comportamiento de los distintos grupos se explican, primero, por la desagregación en subgrupos en los que se supone que todos los individuos muestran un comportamiento uniforme y, segundo, por una consolidación en la cual cada subgrupo se pondera apropiadamente para reflejar su participación cambiante en el agregado total. Asi, los patrones de mortalidad se explican por las distintas composiciones de edad, sexo, y la tasa de mortalidad por causas específicas; el comportamiento de la fecundidad se explica por los distintos patrones de edad al casarse, tamaño de familia deseado y espaciamiento de los nacimientos.

Por otra parte, la perspectiva del economista en la investigación, está dirigida por lo general a la explicación de la elección que efectúan los individuos y los establecimientos. Las decisiones en relación con lo que se produce, cómo, para quién y con cuáles consecuencias, representan el punto central de la óptica de los economistas. Según algunos asentamientos de mercado, por ejemplo, se supone que la maximización de la satisfacción de los consumidores interacciona con la maximización de la utilidad de los productores, con el objeto de determinar un conjunto de equilibrio entre precios y cantidades. En este sentido es que las decisiones económicas están ligadas a supuestos agentes independientes.

Finalmente, existe la perspectiva de investigación del geógrafo. La primera ley de la geografía afirma que "todo se relaciona con el todo a su alrededor, pero las cosas más cercanas están más relacionadas que las cosas distantes" (Tobler, 1970). Por lo tanto, la perspectiva principal de esta disciplina es: que lo que sucede en un lugar determinado está ligado con lo que sucede en los lugares adyacentes. Es el panorama de la interdependencia espacial de todos los fenómenos el que se refleja en las explicaciones de los patrones de urbanización y desarrollo.

Los demógrafos, economistas y geógrafos han contribuido al estudio del proceso de urbanización y desarrollo, asi como de los problemas a los que se ha enfrentado el IIASA. Cada grupo de estudiosos, sin embargo, ha buscado la identificación de los determinantes y consecuencias principales, tal como lo han dado a conocer en las perspectivas de su disciplina en particular. Se han congregado en el Instituto en la creencia de que la combinación de los diferentes puntos 
de vista dentro de los mismos procesos sociales, pueden contribuir al logro de descubrimientos importantes para comprender los efectos de la urbanización y su administración.

\section{Urbanización: la versión del demógrafo}

Los demógrafos han interpretado las tasas de crecimiento y urbanización aceleradas predominantes en la actualidad en los países de menor desarrollo, como las consecuencias directas del incremento en las tasas de aumento natural (nacimientos menos muertes), y las tasas netas de migración urbana (inmigración urbana menos emigración urbana). Las variantes temporales y espaciales expuestas por estos dos conjuntos, han seguido generalizaciones convencionales y descriptivas que son de interés para explicar las regularidades históricas descritas como "transiciones" o "revoluciones". En esencia, el crecimiento de la población se atribuye a la revolución vital (o revolución demográfica), proceso por el cual las sociedades con tasas elevadas de natalidad y mortalidad se dirigen a una situación de tasas bajas de natalidad y mortalidad en el curso del desarrollo. La urbanización se explica como un resultado de la revolución de movilidad - la transformación que han experinentado las sociedades con tasas bajas de migraciónmientras avanzan a la condición de tasas elevadas de migración. Estas dos revoluciones se llevan a cabo de manera simultánea y articulada para dar lugar a la transición demográfica: la versión clásica del demógrafo sobre la población y el desarrollo (gráfica 2).

La urbanización es el resultado derivado de una interacción espacial, principalmente de las revoluciones vital y de movilidad. Se caracteriza por distintos niveles de mortalidad urbana - diferenciales rurales en la fecundidad-y el patrón de descenso, además de la transferencia neta masiva de la población de las áreas rurales a las urbanas a través de la migración interna, genera asi un proceso de urbanización por el que atraviesan todas las naciones en el curso de su transición de una sociedad agrícola a una industrial. Estos patrones de urbanización pueden mostrarse por una curva en forma de S alargada (gráfica 3). De manera típica muestran un aumento, repentino de cerca del 20 por ciento, se estabiliza en algún punto entre 40 y 60 por ciento, para detenerse, o hasta lograr un descenso en la proporción urbana, en los niveles cercanos al 75 por ciento.

\section{Desarrollo: la versión del economista}

Los principales aspectos que caracterizan la versión del economista sobre la urbanización y el desarrollo, son:

-Tasas elevadas del ingreso per cápita o del crecimiento del producto (15-20 por ciento por decenio).

- Tasas relativamente elevadas del crecimiento de la población.

-Un descenso agudo (cerca del 10 por ciento por decenio) en la importancia relativa del sector agrícola. 
Grạ́fica 2

LA TRANSICIÓN DEMOGRÁFICA

Revolución vital

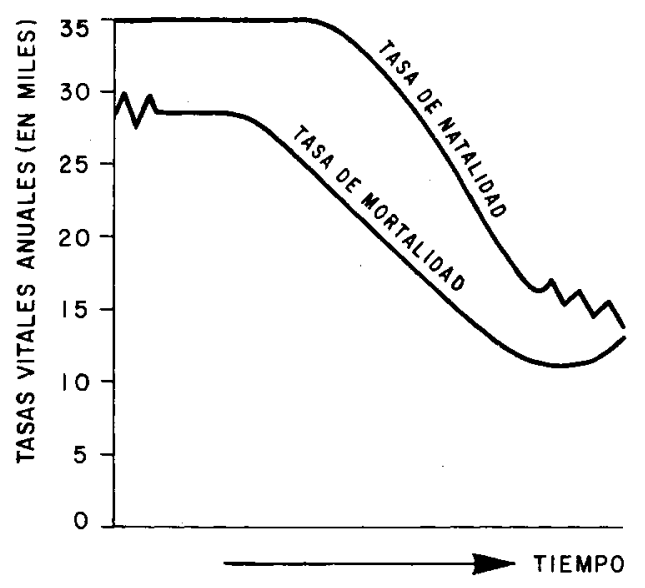

Revolución de movilidad

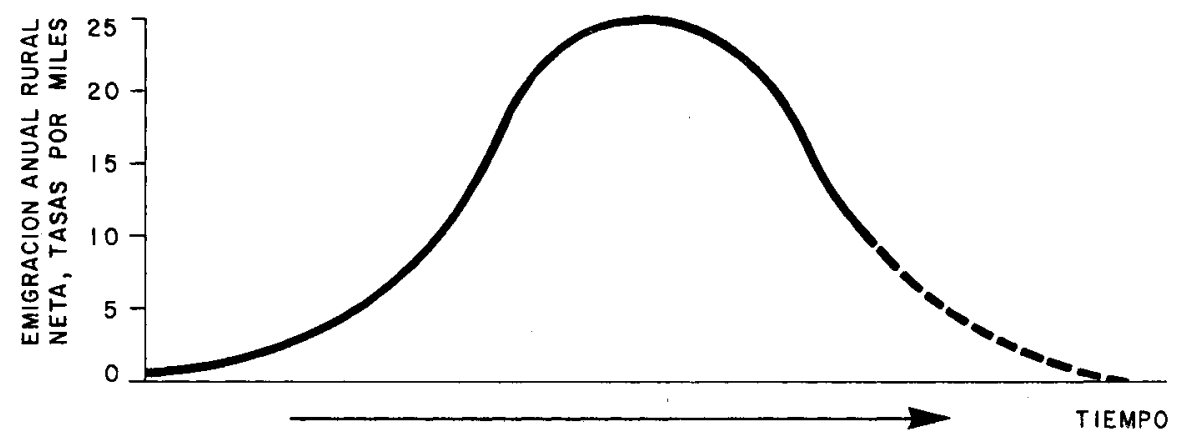

FUENTE: Berelson (1973) y Zelinsky (1971) 


\section{Gráfica 3}

EVOLUCIÓN HISTÓRICA DE LA PROPORCIÓN DE UNA POBLACIÓN CLASIFICADA COMO URBANA.

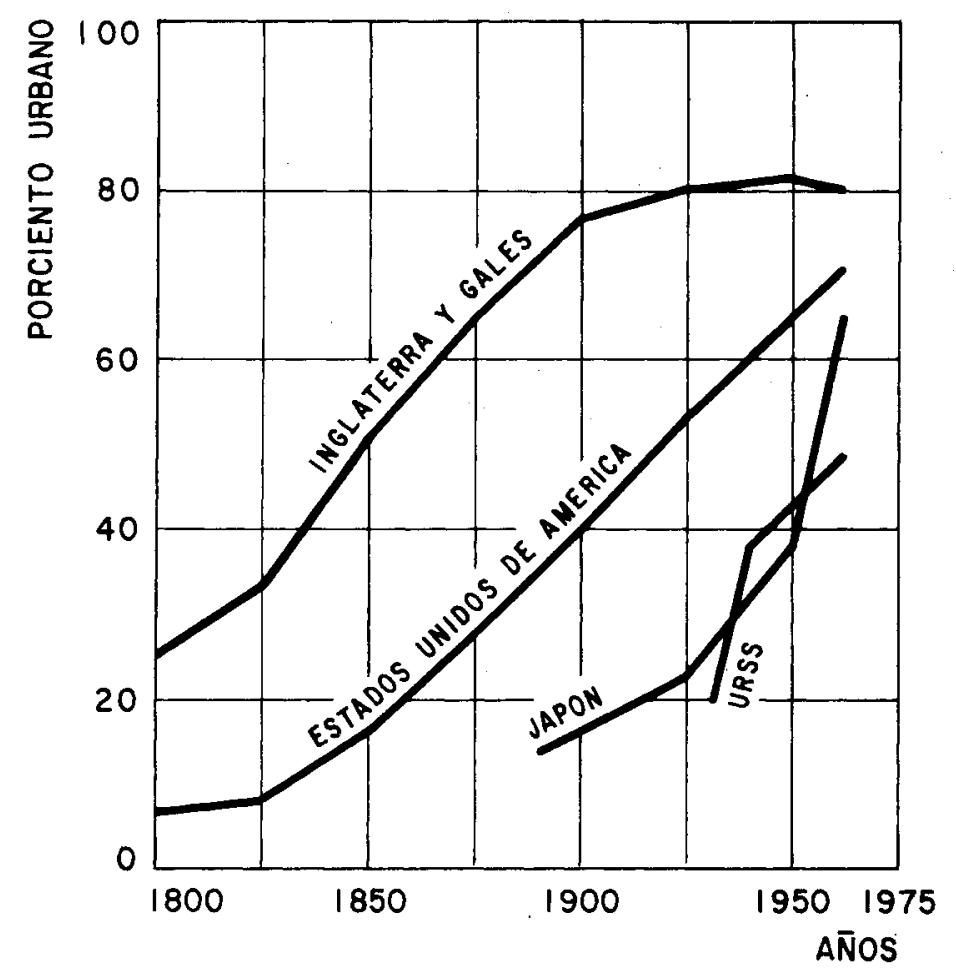

FUENTE: Davis (1965).

El descenso en la fracción relativa de la fuerza de trabajo que participa en la agricultura que acompañó al crecimiento económico en el mundo occidental, por lo general se explica por las fuerzas paralelas de la demanda más baja de alimentos y productos agrícolas comparada con otros productos y los efectos de la tecnología sustitutiva de mano de obra en la agricultura. Los cambios estructurales orientados hacia los sectores no agrícolas se hacen evidentes en los cambios de la participación sectorial del producto nacional y la composición del trabajo industrial asociado. La participación de la fuerza de trabajo agrícola tiene un descenso del 80 por ciento en la economía preindustrial, a cerca del 10 por ciento en etapas más avanzadas del desarrollo. En relación con este descenso, se dan aumentos de la participación de los sectores secundario y terciario: industria y servicios (gráfica 4). 
Gráfica 4

LA TRANSFORMACIÓN ESTRUCTURAL: CAMBIO DE LOS PORCENTAJES DE POBLACIÓN EN LA AGRICULTURA, INDUSTRIA Y SERVICIOS, COMO FUNCIÓN DEL INCREMENTO DEL INGRESO PER CÁPITA

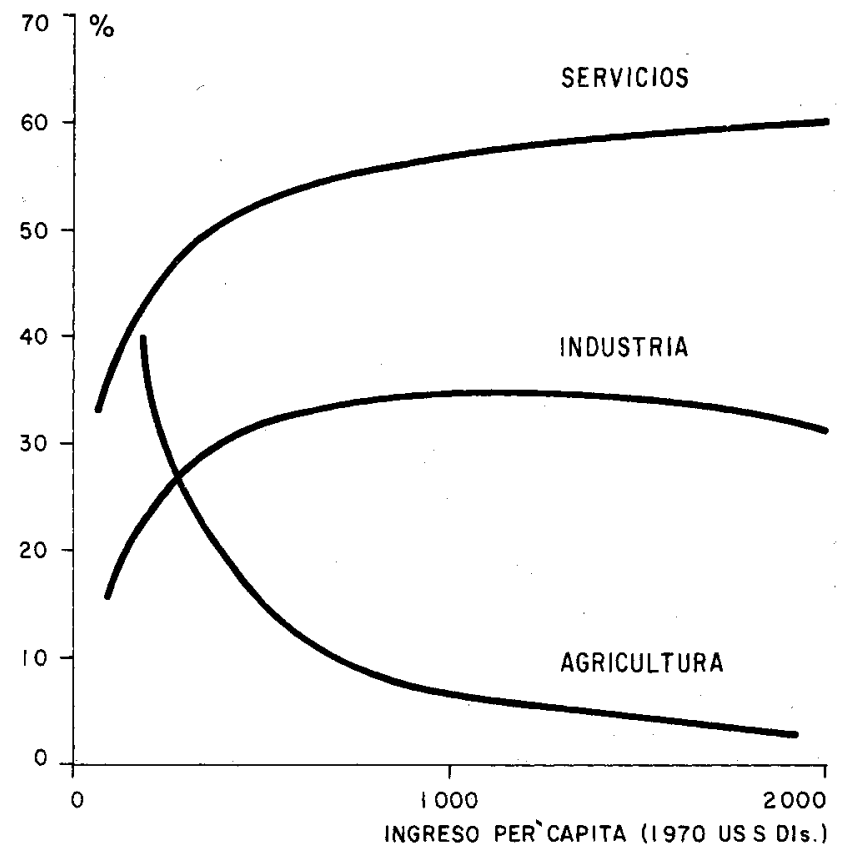

FUENTE: Chen (1979)

La transformación estructural de una economía es una correlación importante en la redistribución espacial concomitante de su fuerza de trabajo, y de su población fuera de los asentamientos rurales y hacia los urbanos (gráfica 5). La urbanización en los países desarrollados estuvo asociada con la industrialización; la urbanización en los países de menor desarrollo de la actualidad supera a la industrialización, lo que ha llevado a algunos estudiosos a llamar a tales paises "sobreurbanizados".

\section{Asentamientos: la versión del geógrafo}

Los geógrafos han examinado los patrones de urbanización y desarrollo de una manera un poco distinta. Argumentan, tomando una visión global de los patrones nacionales de asentamientos, que las regularidades en la distribución del tamaño de las ciudades, reflejan la naturaleza del crecimiento y disminución del sistema nacional de ciudades y del proceso de urbanización. Piensan, que en cada país se han organizado los centros urbanos de manera funcional dentro de 
Gráfica 5

GRADO DE URBANIZACIÓN EN COMPARACIÓN CON EL PNB PER CÁPITA

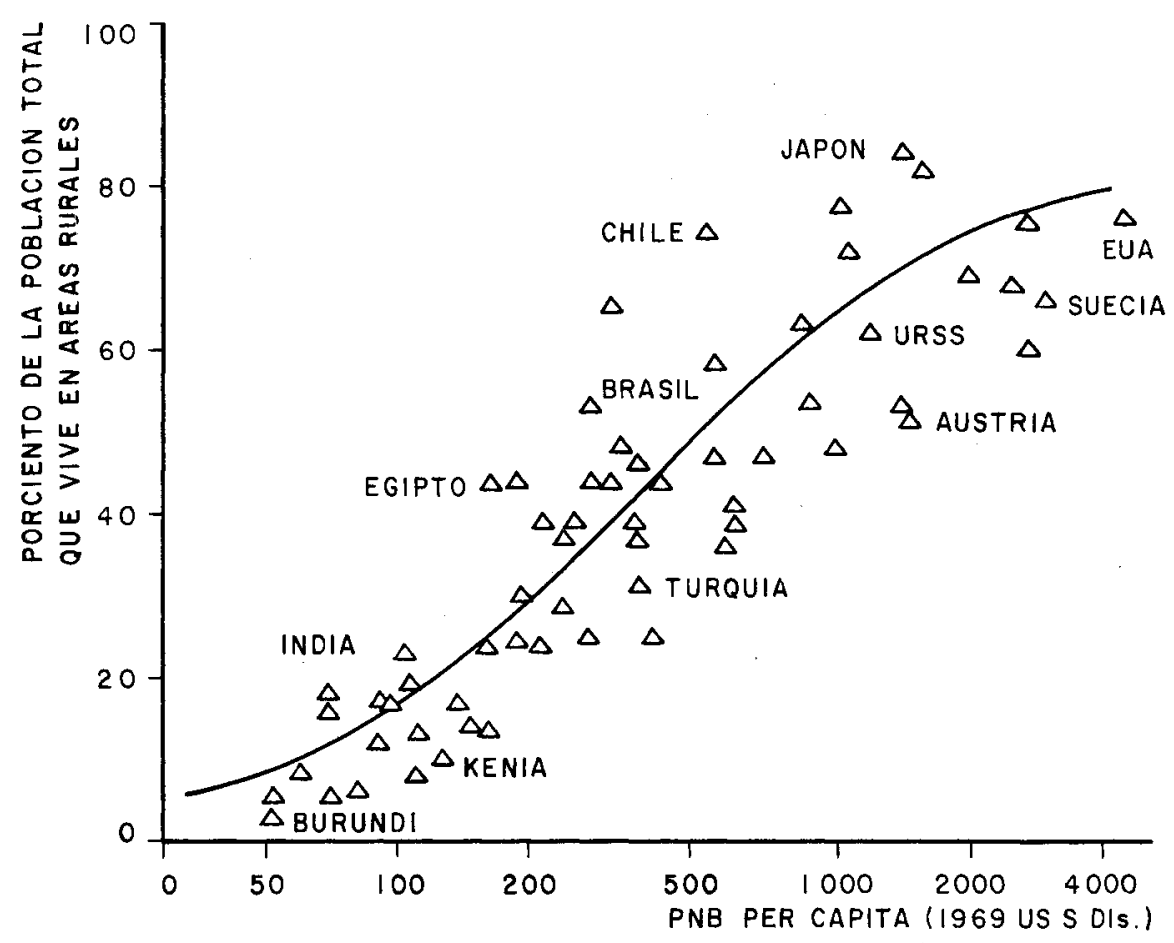

FUENTE: Reproducción de la gráfica de Berry (1973: 1975)

una jerarquía que refleja la integración espacial de la economía nacional. E1 tamaño de la población es el índice más simple de la posición de la ciudad en tal jerarquía, y las diferencias en las cifras de las ciudades en los diversos tipos de tamaño revelan diferencias de organización jerárquica. Esta organización, a su vez, se relaciona con las diferencias en los patrones de urbanización y desarrollo (Hansen, 1978; Sheppard, 1980).

Los geógrafos han mostrado que una distribución de tamaño en particular surge de un proceso de crecimiento en el cual el aumento de las ciudades es proporcional a su tamaño. Más aún, algunos han asegurado que, cuando prevalece tal patrón de crecimiento, la frecuencia de distribución que se desarrolla es el conocido rango-tamaño, o distribución lognormal (Berry, 1964 y 1973). Si esta última distribución fuera verdadera, entonces la gráfica de los porcentajes acumulados de las ciudades a partir de un umbral mínimo -localizado a lo largo de una escala normal de probabilidades contra el logaritmo de tamaño de ciudadda lugar a una línea recta. 
Las desviaciones sistemáticas de la ciudad más grande o de ciudades de población esperada dadas por la ley rango-tamaño arrojan un ascenso hacia un diagnóstico de "preeminencia". Tailandia es un ejemplo representativo en virtud de que Bangkok, su ciudad con mayor número de habitantes, es casi cuarenta veces más elevada que la segunda ciudad más grande (gráfica 6).

Las regularidades en la forma de distribución lognormal se han asociado con los sistemas urbanos de los países de mayor desarrollo. Las distribuciones preeminentes, por otro lado, se han asociado con los países de menor desarrollo. El

\section{Gráfica 6}

\section{DISTRIBUCIÓN DE TAMAÑOS DE CIUDADES}
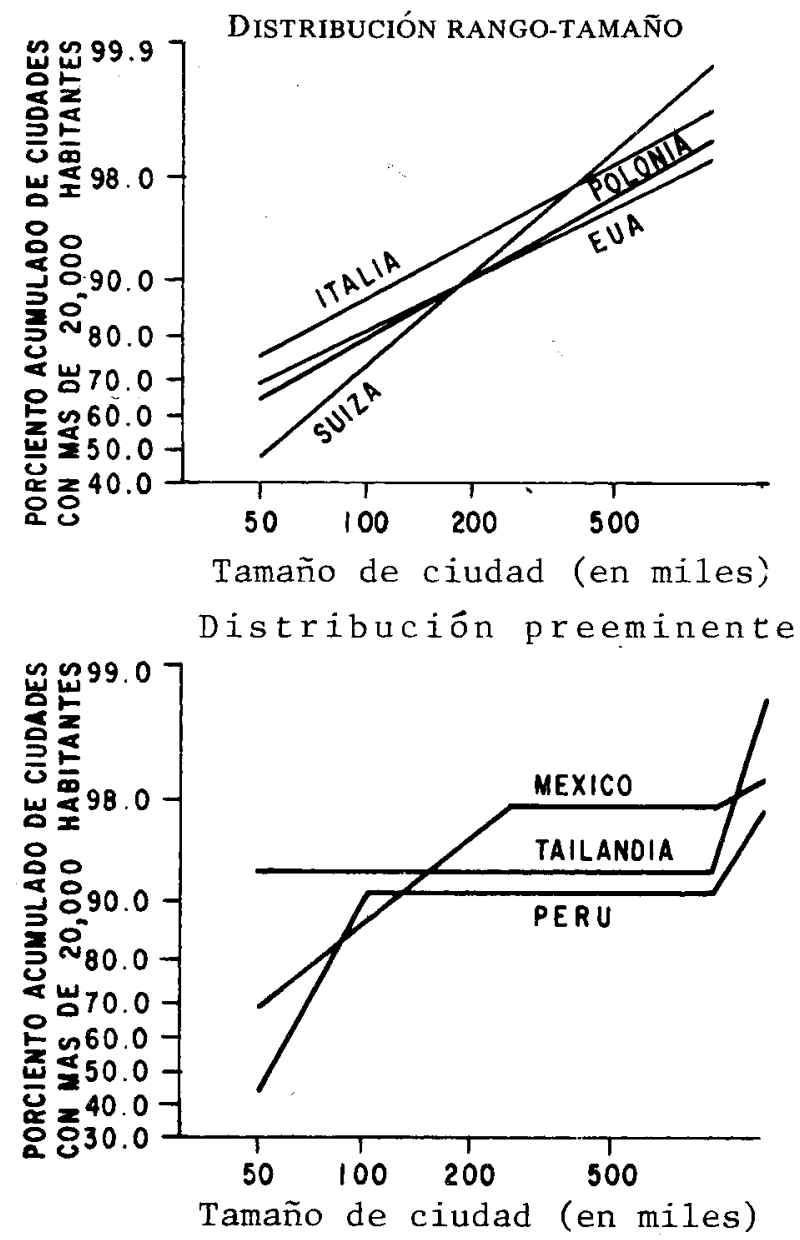

FUENTE: Berry (1961) 


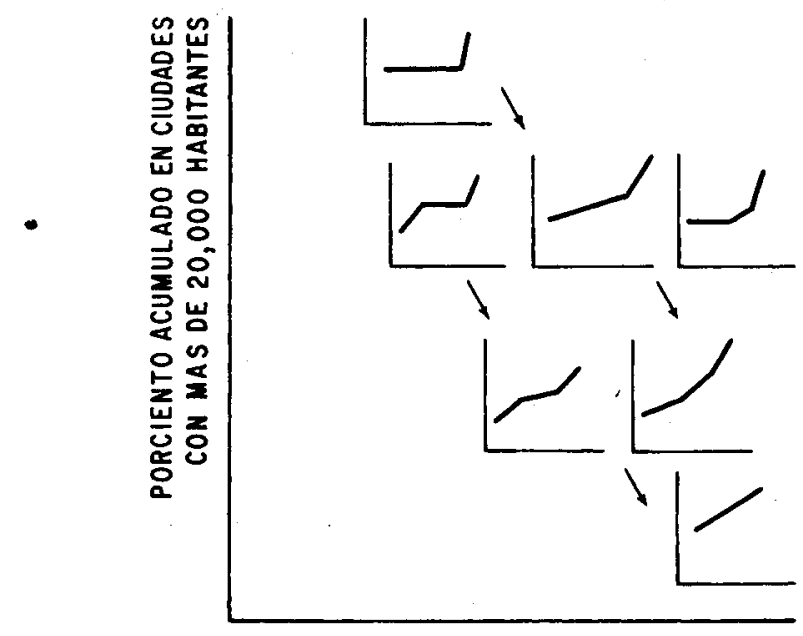

TAMAÑO DE LA CIUDAD (EN MILES)

FUENTE: Berry (1961)

examen de la distribución histórica del tamaño de las ciudades ha llevado a algunos geógrafos a colocar el proceso evolutivo en donde la distribución preeminente se transforma, de manera gradual, en una distribución lognormal a lo largo del desarrollo nacional (gráfica 7).

Crecimiento de la población, cambio estructural, interacción espacial: versión interdisciplinaria del IIASA

Durante los últimos años, los demógrafos, economistas y geógrafos han contribuido a la elaboración de las versiones del IIASA en la dinámica de la urbanización, desarrollo y asentamientos humanos. A pesar de que la verdadera perspectiva interdisciplinaria todavía se encuentra en vías de formación, ya se ha logrado un avance importante en el enlace multirregional de los modelos demográficos de sobrevivencia de cohortes con un modelo económico de equilibrio general multisectorial, y con modelos geográficos multilocacionales de interacción espacial.

La función principal del modelo de simulación demoeconómico de urbanización y desarrollo, es la de averiguar la importancia cuantitativa de los efectos indirectos de los cambios en el medio demográfico o económico. La importancia fundamental de esta interdependencia en los modelos demoeconómicos conduce, 
de manera natural, al uso de enfoques de equilibrio general en análisis teóricos y empíricos. Mientras los modelos de equilibrio parcial han enfocado de manera ventajosa la operación de varios componentes del proceso de crecimiento económico, éstos no pueden asociarse, por su misma naturaleza, con la interdependencia y retroalimentación que son características del proceso de cambio estructural.

Durante los últimos dos años, el IIASA ha avanzado en el diseño de un modelo de equilibrio general de desarrollo demoeconómico. El propósito de la elaboración de tales modelos ha sido proporcionar estructuras analíticas y marcos empíricos para analizar los determinantes y consecuencias de los cambios estructurales y de urbanización. Estos modelos ponen de relieve las conexiones de la demanda de servicios de la población, así como la sustitución tanto en el consumo como en la producción. Es su deseo contribuir al debate sobre las estrategias a seguir a largo plazo, en la urbanización y en el desarrollo. Además, están diseñados para que puedan ponerse en práctica.

Las ideas que intentan transmitir los modelos del IIASA son:

-urbanización

- distribución del ingreso

-crecimiento y cambio estructural

- demografía y desarrollo

Además de estos temas fundamentales, los modelos también han sido diseñados para integrar algunos de los temas de investigación de IIASA, tales como la satisfacción de necesidades básicas del ser humano (alimentación, salud, educación y vivienda) y los recursos (energía, tierras). También incluye al sector gubernamental que es el que establece los lineamientos políticos con sus poderosos instrumentos: impuesto, consumo y políticas reguladoras. Aun cuando los modelos, en esencia, son de equilibrio general, pueden incorporarse características de desequilibrio y retraso.

Finalmente, los modelos del IIASA sirven de escenario para la elaboración de modelos diseñados para utilizarse en el llamado desarrollo de simulaciones contrafactuales, es decir, simulaciones del tipo "qué pasaría si". Si se quiere averiguar la forma en la cual dos políticas diferentes de crecimiento económico han influido sobre los factores económicos y demográficos, estos modelos pueden correrse con distintos conjuntos de supuestos, de acuerdo con las políticas nacionales de crecimiento económico y el impacto de los factores exógenos que no se incluyen en los modelos.

\section{Perspectivas en el uso de politicas: comprensión de los temas de discusión $y$ posibles alternativas}

Los estudiosos y los diseñadores de políticas con frecuencia no se ponen de acuerdo cuando se trata de valorar la conveniencia de mantener las tasas actuales de urbanización elevada y migración masiva rural-urbana en los países de menor desarrollo. Algunos, los "optimistas", observan estas tendencias como una forma de acelerar el proceso nacional de desarrollo socioeconómico; los otros, los "pesimistas", piensan que las consecuencias de este proceso no son nada deseables y argumentan que ambas tendencias deberían llevarse a cabo de manera más lenta. 
Los que se encuentran del lado negativo argumentan que la mayoria de los paises en desarrollo están "sobreurbanizados" porque las tasas de crecimiento urbano se encuentran rezagadas en relación con las tasas de desarrollo industrial y crecimiento económico. Esto ha originado una desproporción que ocasiona que algunas ciudades de los países de menor desarrollo se encuentren constantemente en crisis.

Los que apoyan las normas actuales de urbanización y migración de los países de menor desarrollo, señalan los beneficios que la modernización trae a la urbanización y los aumentos en el bienestar de la mayoría de los migrantes rural-urbanos. Sostienen que la urbanización transforma la cosmovisión y el comportamiento de los habitantes a la vez que aumenta sus experiencias. También argumentan que la preocupación por el bienestar muy probablemente se encuentra fuera de lugar, porque, a pesar de la inseguridad en el empleo y de las sórdidas condiciones de vida, la mayoría de los migrantes rural-urbanos mejoran su posición. La transferencia del campo a la ciudad les permite aumentar su ingreso personal y obtener servicios sociales más amplios y de una calidad superior a los que tenían en su lugar de origen.

Las tres estrategias a largo plazo relacionadas con la urbanización, con frecuencia proponian que se abordaran los problemas urbanos de los países de menor desarrollo, a saber: reducción de la fecundidad y migración, desarrollo económico y redistribución de la población urbana fuera de las grandes ciudades. En un esfuerzo por lograr una mejor comprensión de la efectividad de tales estrategias, las investigaciones del Instituto en cuanto a migración, urbanización y desarrollo comenzaron a concentrarse en el estudio demográfico de los tres problemas siguientes, todos ellos importantes:

- La fecundidad y migración rural-urbana elevadas son la causa de las tasas elevadas de urbanización y crecimiento urbano que prevalecen en la actualidad en los países de menor desarrollo. ¿Cuál de estos dos componentes del movimiento de población debe recibir mayor atención en las políticas nacionales de población?

- ¿Puede ser la estrategia de industrialización acelerada un modelo adecuado -de inclinación predominantemente urbana - para la mayoría de los países en desarrollo? ¿Deben tener los programas de desarrollo agrícola y rural una participación mucho más amplia de la que tienen en la actualidad?

- ¿Resultan las ciudades más grandes en los países de menor desarrollo muy grandes, además de consumir una participación muy desproporcionada de los recursos nacionales y servicios? ¿No será éste más bien un problema de mala administración del crecimiento urbano que de tamaño de ciudad?

Se han estudiado estas tres preguntas y se han elaborado conclusiones tentativas. Sin embargo, en virtud de que ha evolucionado nuestra investigación interdisciplinaria en los procesos de urbanización y desarrollo, hemos tomado plena conciencia de que tales preguntas tienen grandes limitaciones y son de carácter disciplinario. Cada disciplina académica que trata los problemas del desarrollo urbano, con gran frecuencia observa solamente una visión parcial del problema y se concentra en las politicas conectadas específicamente con ese tema. Por lo tanto, los demógrafos buscan aliviar los problemas del crecimiento urbano por medio de políticas dirigidas a reducir la fecundidad y la migración. Los economistas, por otro lado, al 
abordar tal fenómeno, apuntan sus consideraciones hacia las inversiones adecuadas y las políticas fiscales y monetarias. Lo geógrafos se abocan al reacomodo del patrón de asentamientos humanos.

Sin embargo, estos puntos de vista parciales dan como resultado perspectivas poco adecuadas para diseñar una política nacional de desarrollo urbano. Por ejemplo, varios estudiosos han destacado los impactos territoriales no intencionados de los programas de gobierno, tales como la construcción de carreteras y leyes que regulan el tratamiento de la depreciación y las ganancias sobre capitales (Alonso, 1972). Otros han hecho hincapié en la necesidad de implementar un desarrollo multirregional coordinado con los sectores urbano y rural con el objeto de adoptar estrategias de desarrollo que puedan remediar las desproporciones urbano-rurales (Edwards, 1974). También se ha dirigido la atención, en los actuales países de menor desarrollo a las importantes relaciones que conectan los sectores tradicional y moderno de los mercados de trabajo urbano (Rempel, 1979).

Los distintos programas gubernamentales, las regiones geográficas y los sectores económicos son, todos, piezas eslabonadas en la evolución del rompecabezas que es el desarrollo urbano, por lo que ninguno debe considerarse de manera aislada. El reconocimiento de que "todo está conectado con todo lo demás" no le permite al analista más alternativa que abordar la complejidad de problemas de los sistemás multidimensionales cuando se generan recomendaciones de politicas para elaborar estrategias nacionales de desarrollo urbano. Más aún, los objetivos de tales estrategias deben reflejar todas las políticas nacionales: seguridad, estabilidad, calidad ambiental, además de crecimiento económico, eficiencia e igualdad. Asi, y a modo de ejemplo, en las políticas de desarrollo urbano y de política internacional no pueden identificarse ni adoptarse conjuntos simplistas y unificados de lineamientos operacionales; las consideraciones centrales deben aplicarse dentro del contex to general de objetivos nacionales más amplios antes de formular estrategias efectivas (Alonso, 1972).

Como se ha incrementado la comprensión de la compleja multidimensionalidad de los problemas que van asociados a la urbanización y al desarrollo, nuestras perspectivas en el diseño de políticas se han vuelto más modestas en sus alcances. En la presente etapa de evolución de nuestro trabajo, hacemos dos preguntas fundamentales: ¿descenderán en el futuro las tasas de crecimiento urbano en los países de menor desarrollo - por ejemplo, la ciudad de México tendrá 31 millones de habitantes para el año 2000-? ¿Qué tipo de análisis puede contribuir a resolver algunos de los problemas de administración más acuciantes asociados con tales tasas de crecimiento? Se están utilizando los modelos demoeconómicos del IIASA para ayudar a contestar ambas preguntas.

\section{¿Se reducirá el crecimiento urbano en los paises de menor desarrollo?}

La investigación efectuada en el IIASA indica que continuarán las tasas explosivas de crecimiento urbano en los países de menor desarrollo de la actualidad, probablemente durante algún tiempo y se prevé que, el crecimiento urbano reducido tendrá lugar una vez superada la etapa de transición del desarrollo urbano, en cada uno de los países en desarrollo. 
Los modelos demográficos multirregionales del IIASA sugieren que el crecimiento de las ciudades es, en parte, autolimitante, dado que las tasas de crecimiento urbano han demostrado cierto descenso, mientras que las proporciones urbanas han aumentado y la población rural ha disminuido su crecimiento. Los modelos demográficos recurren a las fuerzas de la transición demográfica para reducir las tasas de crecimiento de las ciudades en el futuro. Las fuerzas de "freno" de estos modelos - por ejemplo, las tasas de fecundidad más bajas de las poblaciones urbanas- requieren un horizonte de tiempo relativamente largo para mostrar un impacto importante (Rogers, 1978). Por otro lado, las fuerzas económicas, tales como el incremento del costo de vida urbano, pueden actuar para disminuir cuanto antes la migración hacia las ciudades y retardar el crecimiento urbano. El prototipo de modelo demoeconómico adoptado por el IIASA (Kelly y Williamson, 1980 ) introduce una retroalimentación potencial de los diversos aumentos de los costos de las ciudades en las decisiones de la migración rural-urbana. Se internaliza en algunas de las fuerzas principales que se pusieron en movimiento para intentar controlar la tasa de urbanización, y son:

- cambios en los costos de los diferenciales de subsistencia entre las áreas urbanas y rurales:

-cambios en la calidad de los bienes públicos de la ciudad y los servicios;

-cambios en los requerimientos de insumos del sector moderno de producción y la escasez de los recursos;

-cambios en las demandas de la acumulación "no productiva" del capital urbano.

Estas fuerzas endógenas generan "límites al crecimiento urbano" en el modelo de urbanización y desarrollo del IIASA. Sin 'embargo, hasta el momento no se ha medido su impacto de manera cuantitativa, puesto que la aplicación de los estudios de caso de tales modelos se empezaron en época reciente.

\section{Administración de la absorción urbana}

La contribución de los analistas de sistemas al diseño de políticas urbanas puede ayudar a lograr un mejor entendimiento de los niveles probables de demanda futura de recursos, empleos, vivienda y servicios durante este período de transición - $\mathrm{y}$ en el posterior-, si es verdad que el paso acelerado del crecimiento urbano de la actualidad en los países de menor desarrollo es una etapa transitoria en el desarrollo urbano. El gran reto que tienen que enfrentar los planificadores y administradores urbanos en las ciudades de los países de menor desarrollo, consiste en absorber de manera efectiva y justa el número elevado de personas que llegan a la ciudad, mientras desarrollan una adecuada política de capacidad de absorción urbana. Los modelos de simulación demoeconómica representan un papel importante para lograr estos esfuerzos, porque pueden utilizarse para delinear las posibles consecuencias de políticás alternativas en los patrones de oferta y demanda.

La gente demanda recursos y servicios, de ahi que si todo lo demás está organizado (incluyendo gustos y precios), el nivel de demanda debe ser proporcional, de manera aproximada, al tamaño de la población. Si la demanda supera este nivel, esto puede atribuirse a la afluencia. 
La asociación entre el consumo de energía y la afluencia se muestra de manera más explícita en la gráfica 8 , que marca el consumo de energía per cápita contra el ingreso per cápita, en 96 naciones del mundo. La correlación es impactante: mientras se lleva a cabo el desarrollo de un país pobre, se requiere una mayor cantidad de transumos de recursos energéticos para sacar adelante su economía y abastecer las necesidades de la población.

Puede obtenerse una primera aproximación satisfactoria de los determinantes demográficos y económicos del nivel y composición de la demanda, al considerar exclusivamente los cambios en el tamaño de la población y su ingreso. Sin embargo, con el fin de obtener un avalúo más completo del impacto de las distintas tendencias poblacionales en la demanda de recursos y servicios, es necesario ir más adelante y examinar los efectos de los cambios en la composición por edades de

\section{Gráfica 8}

RELACIÓN ENTRE EL USO DE ENERGÍA Y EL PNB PER CAPITA DE 26 NACIONES (CIFRAS DE 1965)

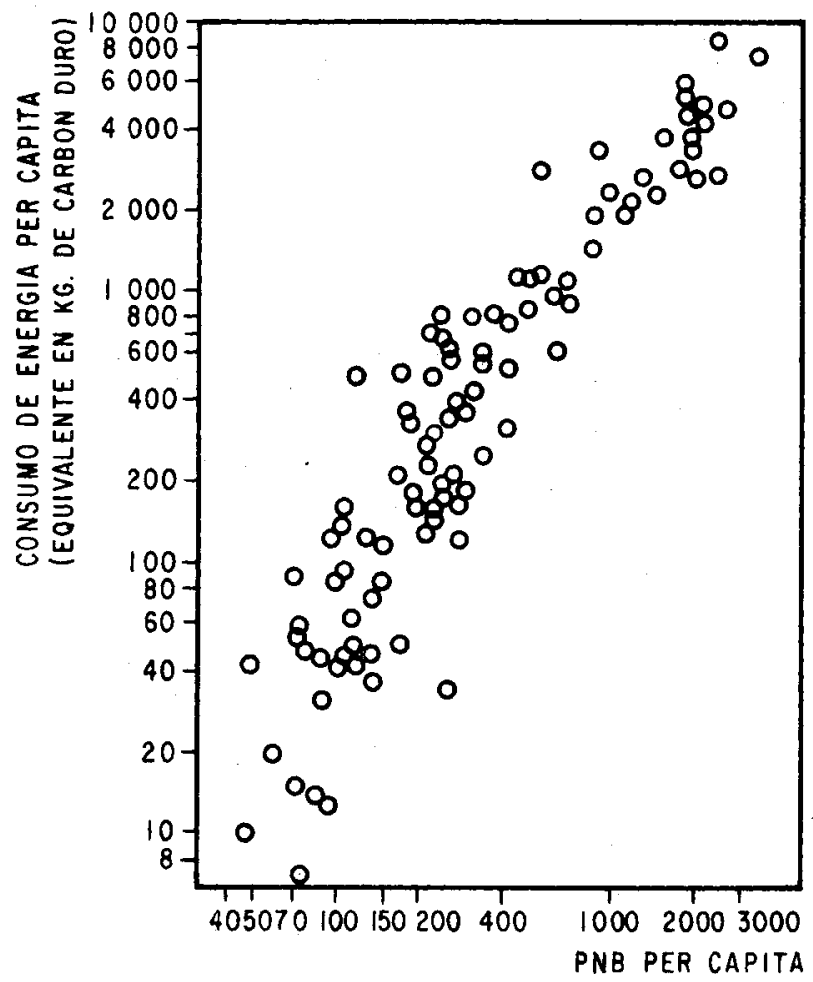

FUENTE: Fisher y Potter (1971). 


\section{Gráfica 9}

\section{RELACIÓN ENTRE EDAD Y DEMANDA DE BIENES Y SERVICIOS}

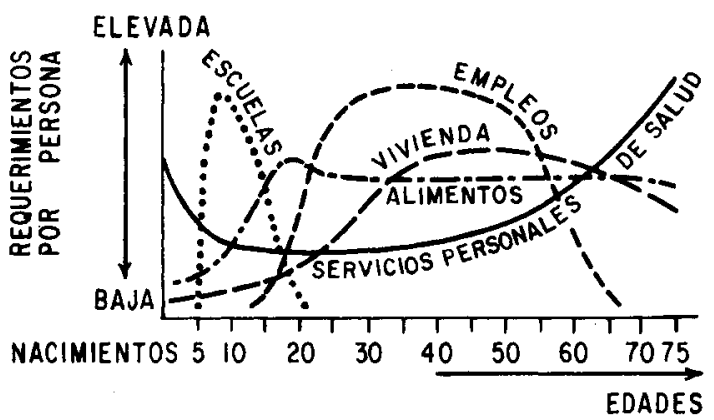

FUENTE: Corsa Jr, y Oakley (1971).

la población en tales demandas. La gráfica 9 ilustra las relaciones entre la composición de edades y la demanda de ciertos servicios. Por ejemplo, esta información muestra que la demanda de servicios educacionales se lleva a cabo, por lo general, entre los 5 y 20 años de edad, para alcanzar el máximo a los 10. La demanda de empleos en la etapa de participación en la fuerza de trabajo se presenta entre los 15 y 65 años. La demanda de servicios de salud es relativamente elevada en los niños pequeños y adultos mayores.

Se debe añadir a las variables del tamaño de población, ingreso per cápita y composición por edades, el lugar geográfico y, en particular, la distinción entre los lugares de residencia urbanos y rurales. La información de la gráfica 10 corresponde a Japón, donde aparecen con claridad los patrones de cambio en el consumo de alimentos efectuado durante el curso del desarrollo y urbanización de la nación. Para cada uno de los tres años que se muestran, la fracción del total de gastos familiares desciende en el tiempo y es más bajo para las familias urbanas en relación con las rurales (campesinas). Más aún, la fracción del total de gastos que se destina a alimentos que contienen almidón (cereales, papas, etc.), también resulta baja para la población urbana, la que tiende a consumir mayores cantidades de alimentos con proteínas animales, tales como carne, lácteos, huevos y pescado.

Finalmente, la demanda de infraestructura urbana y servicios no es una función exclusiva de los residentes de las ciudades; también es una función de un número de usuarios industriales y comerciales. El especial esto se aplica a los servicios públicos, tales como el suministro de agua, electricidad y alcantarillado. Así, la industrializeción genera su propio aumento de demanda en la infraestructura urbana.

En resumen, la utilidad de los modelos demoeconómicos de urbanización y desarrollo del IIASA reside en su contribución para lograr una mejor comprensión de los procesos subyacentes al crecimiento y cambio, además de su capacidad para evaluar los niveles probables de demanda futura de los diversos bienes, ser- 
Gráfica 10

Porcentaje DEl total DE Gastos POR FAMILIA DESTINADOS A GRUPOS ESPECÍfICOS DE ALIMENTOS, EN LAS FAMILIAS URBANAS Y CAMPESINAS EN JAPÓN

A. FAMILIAS URBANAS

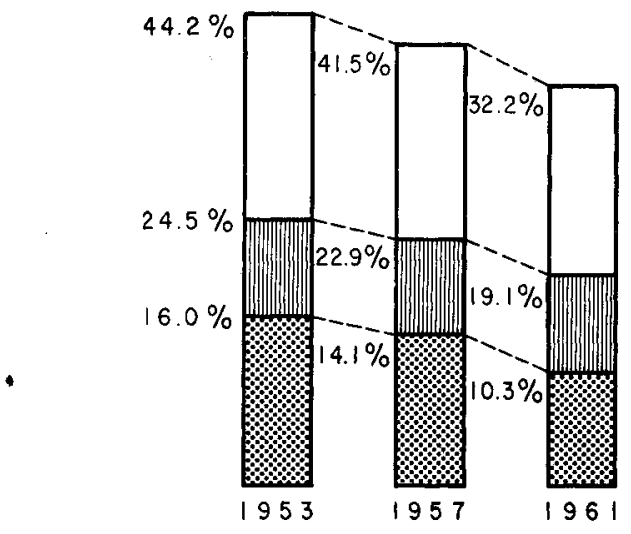

B FAMILIAS CAMPESINAS

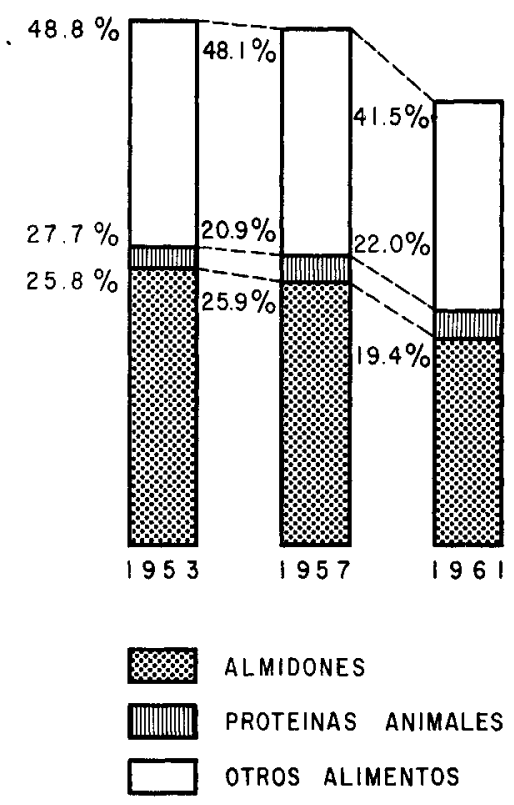

FUENTE: Kaneda (1970) 
vicios e infraestructura. Su característica más importante se basa en la capacidad que tiene para proporcionar una perspectiva multidimensional con una amplia gama de sistemas que tomen en consideración los efectos indirectos probables de las decisiones en las politicas alternativas.

\section{CONCLUSIONES: Qué se ha aprendido con estas perspectivas}

Los fundadores del IIASA aconsejan alcanzar los tres objetivos del Instituto:

- reforzar la cooperación y colaboración internacional,

- lograr un mejoramiento en la ciencia y el arte de lograr un análisis interdisciplinario de sistemas,

- aplicar análisis de sistemas con el objeto de ayudar a resolver los problemas importantes de la humanidad.

Los organizadores de esta conferencia reflejaron tales objetivos en el tema "del problema a la investigación y al uso".

Nuestra investigación sobre los procesos y problemas de urbanización y desarrollo se ha diseñado teniendo estos objetivos en mente. Los ingredientes centrales son: la colaboración internacional, la ciencia interdisciplinaria y los análisis de sistemas aplicados.

La correcta aplicación de análisis es una tarea difícil de llevar a cabo en cualquier disciplina y en cualquier país. Si a esta actividad se añaden dimensiones interdisciplinarias e internacionales la situación se complica enormemente. Sin embargo, el IIASA ha intentado llevarlo a cabo y en algunas instancias los resultados han sido bastante satisfactorios. ¿Qué se ha aprendido en nuestro trabajo sobre urbanización y desarrollo respecto a los procesos de interacción científica que pueda arrojar alguna luz sobre las sugerencias que ayuden a resolver las dificultades y a aumentar los logros?

La enseñanza más importante: reconocer que para lograr algún éxito en los sistemas aplicados, es esencial desarrollar una apreciación de los distintos enfoques sobre la evaluación del problema, el diseño de la investigación y su utilización por parte de los diseñadores de políticas.

\section{Distintas valoraciones del problema: la optimista y la pesimista}

Los debates de política internacional sobre los problemas globales y universales, por lo general contraponen los argumentos de los optimistas a los de los pesimistas. Los primeros aconsejan tomar precauciones y creen que "el tiempo se hará cargo de la situación"; los otros sugieren la necesidad de llevar a cabo intervenciones dramáticas en los procesos sociales y de desarrollo.

Por ejemplo, dentro de la arena de políticas poblacionales, los optimistas argumentan que las tasas elevadas de fecundidad y migración hacia las ciudades, representan una etapa transitoria en la evolución demográfica de las sociedades premodernas a las modernas; los pesimistas señalan la persistencia de creencias tradicionales y al momentum del crecimiento de la población que está incrustado en las poblaciones con tasas elevadas de fecundidad. 
En el debate de las políticas económicas, los optimistas argumentan que la desigualdad en el ingreso disminuirá con el tiempo como consecuencia natural del proceso de desarrollo; los pesimistas señalan el ensanchamiento de la brecha entre ricos y pobres y argumentan que ésta se cerrará únicamente a través de programas efectivos de redistribución del ingreso.

En el debate de políticas geográficas, los optimistas argumentan que la distribución en el tamaño de las ciudades alcanzará un estado de equilibrio armonioso que reflejará la integración espacial con el territorio nacional; los pesimistas aún dudan que la "tamización" que pregonan los optimistas que pugnan por una politica deliberada de inversión para redistribuir las corrientes migratorias, se lleve a cabo.

\section{Distintas perspectivas de investigación: análisis interdisciplinario}

Con anterioridad se describieron las distintas perspectivas de investigación efectuadas por los demógrafos, economistas y geográfos. Esta discusión puede ampliarse de manera considerable si el grupo interdisciplinario de científicos sociales incluye a científicos de las ciencias naturales, ingenieros y matemáticos. Deben tomarse en consideración, por ejemplo, las distintas observaciones que efectúe ese grupo de un problema global o universal que se esté analizando en el IIASA. En algunos campos, como el de la ingeniería, la investigación debe orientarse hacia los problemas prevalecientes y efectuar recomendaciones en cuanto a la forma de resolverlos o mitigarlos, en base a los conocimientos con que se cuentan en la actualidad y no en los del futuro. En otros campos, como el de la economía, la orientación debe dirigirse hacia esa disciplina específica, misma que debe buscar el mejoramiento de su comprensión -como prerrequisito y primer paso nesesarios-, antes de ofrecer alguna recomendación en la elaboración de políticas. Los que se inclinan por este último estilo de investigación, con gran frecuencia se impacientan cuando tienen que apoyarse en los primeros, lo que puede dar como resultado algunos malentendidos.

En relación con estas dos perspectivas existen distintos estilos de modelos: el macro en comparación con el micro y el de desequilibrio frente al de equilibrio. Los que pugnan por ofrecer prescripciones en relación a los sistemas complejos, con frecuencia adoptan un modelo relativamente grande y complejo. Otros, con frecuencia utilizan solamente un modelo para ilustrar una parábola (una narración interesante que revela una enseñanza moral) $y$, por tanto, con frecuencia se contentan con un modelo transparente y pequeño si se encamina hacia el lugar deseado. Los que se inclinan por la segunda perspectiva, es probable que critiquen al diseñador de modelos complejos como si quisiera construir un rascacielos utilizando madera como único material. De nuevo, pueden surgir malentendidos.

Finalmente, algunos analistas de sistemas tienden a concentrarse en cifras y observan su disponibilidad tan limitada como la imposición de un límite al crecimiento de la sociedad. En este sentido, el sistema con frecuencia parece que va a desequilibrarse y dirigirse hacia el desastre si no se le controla. Otros, tienden a concentrarse en los valores relativos más que en las cifras y piensan que la sustitución empujará al sistema hacia un equilibrio. Piensan que un artículo se encarece 
cuando escasea y que, de esta manera, la gente lo utilizará menos, mitigándose la escasez.

Distintos usos de planes y politicas por parte de los planificadores de decisiones

Los análisis de sistemas se han identificado enormemente con la comprensión y la consistencia. Esta identificación ha fomentado el diseño y desarrollo de grandes y complejos sistemas. Por ciertas razones, este tipo de comprensión ha sido fuertemente criticado y se ha sugerido que se adopte en su lugar una forma de análisis de políticas que enlace la inteligencia científica y técnica con la acción social organizada dentro de un amplio rango de problemas. Una cuestión central en este debate ha sido la forma adecuada en que los analistas de sistemas ofrecen la inteligencia científica y técnica.

Algunos analistas de sistemas piensan que los que elaboran las decisiones -ya sea. . . gobernador de una ciudad, el gerente de una corporación, un ministro de salud, el gobernador de un Estado- precisan planes comprensivos, un conjunto de anteproyectos diseñados en base a normas aceptadas y estructuradas por medio de un conjunto de reglamentaciones consistentes. Otros piensan que no existen planes que puedan ser comprensivos en virtud de que las personas tienen distintas normas y que las politicas estructuradas a través de programas e incentivos, son instrumentos de acción social mucho más efectivos. La primera perspectiva puede caracterizarse por estar más preocupada en proyectar los resultados y la forma de influenciarlos; la segunda puede caracterizarse por estar preocupada por examinar las reglas de comportamiento que producen los resultados y la forma en que éstos deben cambiarse.

La contribución principal que pueden aportar los análisis de sistemas a la elaboración de políticas es un entendimiento multidimensional y amplio de los temas y estrategias más pertinentes, con base en un análisis interdisciplinario adecuado del comportamiento de los sistemas en cuestión.

Las tasas de crecimiento de la población y de la economía, así como la distribución geográfica de tal crecimiento por todo el territorio nacional, son aspectos de un todo interrelacionado y complejo: nada menos que la evolución de una sociedad durante su transformación estructural de una economía agraria a una industrial y de servicios. Los proyectos y programas diseñados para modificar cualquier aspecto aislado dentro de esta evolución, para que sea efectivo, debe tomar en cuenta un amplio sistema de interdependencia que caracterice tales procesos. 